\title{
EFFECT OF DIFFERENT FIXED DETACHABLE IMPLANT SUPPORTED PROSTHESIS MATERIALS ON THE STRESSES INDUCED ON THE SUPPORTING STRUCTURES
}

\author{
M. Shady Nabhan*
}

\begin{abstract}
Objectives: This study was conducted to evaluate the effect of different implant supported fixed detachable prosthesis materials (chrome cobalt-acrylic and BioHPP (Bio-High Performance Polyether)) on the stresses induced on the supporting structures.

Materials and methods: A maxillary completely edentulous resin cast was $3 \mathrm{~d}$ printed with four implant sites (two straight anterior implants and two tilted posterior implants), mucosal space for mucosa simulation and vertical slots for strain gauges which were $1 \mathrm{~mm}$ distal to the two distal implants. Four implant analogues were placed in their sites. Multiunit abutments were secured to the implants, then fixed detachable prostheses were fabricated. In this study two models were conducted: Group (A): Complete implant supported chrome cobalt-acrylic fixed detachable prosthesis. Group (B): Complete implant supported BioHPP fixed detachable prosthesis. Strain gauges were supplied with fully encapsulated grids and attached wires. Micro strains were recorded at each site of the strain gauges with enough time elapsed between each testing. The applied load started from 0 up to $100 \mathrm{~N}$. T test was used for comparison between the groups, at a significance level of $\mathrm{P} \leq 0.05$.
\end{abstract}

Results: Group A (chrome cobalt- acrylic prosthesis) showed higher induced stresses on supporting structures compared to group B (BioHPP) during bilateral, unilateral, and oblique loading. Independent $\mathrm{T}$ test showed statistically significant difference between the studied groups, $\mathrm{P}$ value was $<0.05$.

Conclusion: Unilateral loading was more traumatic to the implants as compared to bilateral loading. BioHPP prosthesis has damping effect as it transferred less stresses to the supporting structures compared to chrome cobalt- acrylic prosthesis.

KEY WORDS: fixed detachable prosthesis, all on four, BioHPP, strain gauge.

\footnotetext{
*Lecturer of Removable Prosthodontics, Faculty of Dentistry Ain Shams University.
} 


\section{INTRODUCTION}

The "all-on-four" treatment concept was developed to take full advantage of the use of available bone in severely resorbed jaws, allowing immediate loading, function and avoiding bone augmentation procedures that increase costs and complications ${ }^{(1)}$. The all on four concept uses four dental implants in the anterior part of completely edentulous jaws to support a provisional, screw retained and immediately loaded prosthesis. The two most anterior dental implants are placed axially, whereas the two posterior dental implants are tilted distally to maximize anteroposterior spread, and minimize cantilever length ${ }^{(2,3)}$.

The Brånemark surgical-prosthetic protocol supported the placement of four dental implants for the restoration of a severely resorbed mandible and six dental implants in the lower arch that shows minimal to moderate ridge resorption ${ }^{(4)}$. Immediate loading concept for edentulous jaws have become broadly popular among practioners as well as among patients ${ }^{(5,6)}$. High survival rates and a low incidence of complications reveal the predictability of dental implant treatment, regardless of the loading protocol used $^{(7,8)}$.

Clinical success of dental implants depends mainly on the biomechanical considerations and proper distribution of occlusal load. Proper diagnosis and treatment planning are keys for success of dental implants. In compromised conditions such as poor quality of the bone or quantity, strain to the crestal bone can be reduced by increasing the anteroposterior spread of dental implants, placement of longer or wider implants and increasing the number of implants ${ }^{(9)}$.

Full-mouth rehabilitation of a patient using dental implants is a challenge with a compromised ridge condition (vertical and horizontal bone loss); however, hard- and soft-tissue grafting may allow the implants to be placed into the desired positions. Bone grafting especially vertical bone augmenta- tion has limited success rate and usually requires additional surgical interventions, time and costs. All on four treatment concept provides an alternative to ridge augmentation and complicated surgical procedures in compromised ridge at much less time and cost and with more predictable results. Various material combinations including metal/acrylic, metal/ ceramic, BioHPP and zirconia/ceramic have been used for constructing this type of restoration ${ }^{(10)}$.

Fixed detachable dental prostheses made of metal/acrylic may suffer from the following problems: loss of the acrylic teeth, lack of natural color primarily in the prosthetic gingiva area, plaque accumulation and wear of acrylic teeth. Frequent replacement of teeth and maintenance of the prosthesis are required ${ }^{(11)}$.

Prostheses made of metal/porcelain have an excellent esthetic result; however, a major drawback of metal/porcelain prostheses is porcelain chipping. In zirconia/ceramic prostheses, ceramic chipping or breakage of the zirconia framework are the main disadvantages ${ }^{(12)}$.

BioHPP (High Performance Polymer) is based on polyether-ether-ketone (PEEK) polymer and was presented as a dental material for construction of the superstructure prosthesis on dental implants. Its strength is due to the ceramic filler, which improves the mechanical properties. Due to its very small grain size ( 0.3 to $0.5 \mu \mathrm{m})$, constant homogeneity can be established. This homogeneity is important for these material properties and forms the foundation for constant quality ${ }^{(13) .}$

BioHPP is almost as elastic as bone, which helps dissipate any stresses and decrease stress shielding. This also means bone-related torsion can also be balanced out, which is important with larger implant frameworks. BioHPP is also particularly suitable for allergic patients because of its low water solubility of $<0.3 \mu \mathrm{g} / \mathrm{mm}^{3}$ and its low reactivity to other materials ${ }^{(14)}$. 
BioHPP is a very biocompatible materials and can be used for several applications, e.g., for dental implants, provisional abutments, and fixed dental prostheses (FDPs). It is also used for implant-supported bars and clamps for removable prostheses. Furthermore, recent publications reported that BioHPP is a suitable material for telescopic crowns ${ }^{(13,14) .}$

Strain gauges have been used to study stresses induced in dental structures. They allow in vivo and in vitro measurement of the forces on oral implants and supporting structures. Strain gauges can be used to clarify the biomechanical performance of implant-supported prosthesis simulating the variation of number or inclination of implants thus it can be used to assess the effect of the number of abutments and inclination of distal implants on the axial forces and bending moments in implantsupported prosthesis ${ }^{(15)}$.

So the objective of this study was to assess different implant supported fixed detachable prosthesis materials (chrome cobalt-acrylic and BioHPP) regarding stresses induced on the supporting structures.

\section{MATERIALS AND METHODS}

This stress analysis study was conducted invitro using a 3D model simulating a completely edentulous upper arch with four implants positioned as follow: two axial implants in the anterior region and two distally tilted implants in the posterior region to support complete maxillary fixed detachable prosthesis.

In this study, two prosthesis were conducted:

Group A: implant-supported chrome cobaltacrylic fixed detachable dental prostheses.

Group B: implant-supported BioHPP fixed detachable dental prostheses.

\section{Construction of the 3D model cast:}

A scan of an educational completely edentulous maxillary model was done via desktop scanner (3Shape desktop scanner, Denmark), then an STL file was generated. In this STL file four implants beds were designed representing the sites planned for the four implants with dimensions $3.7 \times 11.5 \mathrm{~mm}$. They were planned to be at equal distance from the midline, two implants placed axially in the anterior region and two distally angulated implants placed posteriorly at 30 degrees. Also, two grooves were designed distal to the posterior implants sites for the attachment of the strain gauge.

These grooves were prepared $1 \mathrm{~mm}$ away from the implants. A 2-mm layer thickness was removed from the scanned model crest, which represented the future mucosa. STL file was 3d printed using DLP 3d printer (Envision tec DDDP, Gmbh, Germany).

A mucosa key index design was made over the scanned model. Its design is similar to a special tray which would closely fit over the model to allow the mimic of the viscoelasticic behavior of the mucoperiosteum covering the residual ridges. Implants analogues were placed in the model by press fitting, then mucosa simulation was done via rubber base material (Multisil-Mask soft, Bredent, senden, Germany). (Fig.1)

Construction of complete implant supported chrome cobalt fixed detachable prosthesis:

Multi-unit abutments were screwed (tightened to $25 \mathrm{Ncm}$ according to manufacturer's recommendations) on implants for enabling proper parallelism between the abutments sleeves. Transfer copings were placed, and were all connected together using acrylic resin. A special tray with open top holes was constructed over the transfer abutments. The tray was then loaded with medium body rubber base Silicone (3M ${ }^{\text {TM }}$ ESPE ${ }^{\mathrm{TM}}$ (Soft) Monophase Polyether Impression Material), and an overall impression was obtained. Gingiva mask material was injected in the impression using Multisil Mask. 
A cast was poured using dental stone and castable plastic abutments were inserted and joined in between by PI-KU plast (PI-KU plast, Bredent) for fabrication of the metal framework pattern, cobalt-chromium (Brealloy F 400 Bredent) metal alloy was cast utilizing the lost wax technique, setting of acrylic teeth extending from the right first molar to the left first molar and including sleeves for abutment screws. Teeth-setting was made by pink modelling wax and waxing up of the prosthesis flanges was done. (Fig.2)

Then the waxed up prosthesis was processed using conventional technique. Finally complete arch implant supported cobalt-chromium-acrylic denture was obtained which was passively screwed on the implants and properly seated on the model.

Construction of complete implant supported BioHPP fixed detachable prosthesis

Implant scan body used was attached to implant analogue for obtaining scannable abutments. Scanning of cast without mucosa simulation layer was done with desktop scanner. Design of prothesis was done using Exocad (Exocad, GmbH, Germany). Anatomic wax-up was made virtually and produced as one unit. Cut back for denture teeth and gingiva was done to allow space for denture teeth veneers and esthetic pink gingiva. Scanning of cast with mucosa simulating layer was done to allow accurate adaptation of the final restoration on the gingiva to prevent making pressure on it.

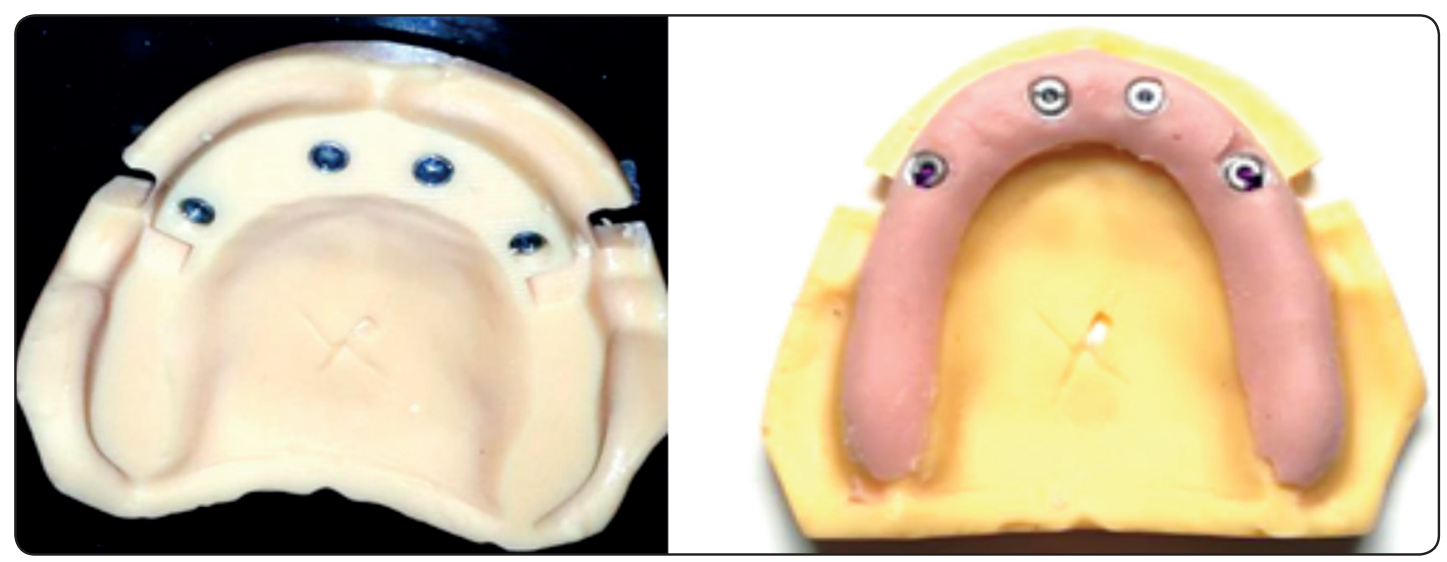

Fig. (1): $3 d$ printed cast with implants and mucosa simulation layer in place.

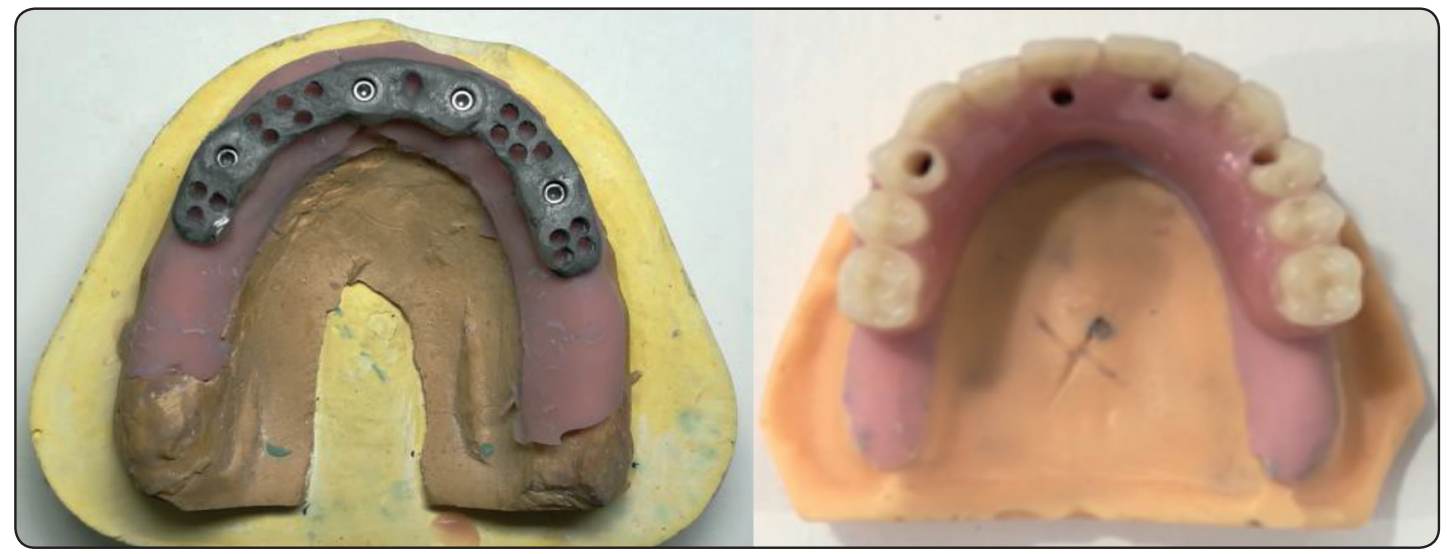

Fig. (2): Construction of chrome cobalt - acrylic prosthesis. 
The final designed restoration was exported as STL file, from which milling of restoration was performed. During milling we block of BioHPP material. BioHPP framework was returned to the cast. Denture teeth veneers (Novo-lign, Bredent, senden, Germany) (novo-lign A44 for anterior teeth and novo-lign G3P for posterior teeth teeth) was used which are made from high-impact PMMA composite, were set and adhered to the framework by wax (Beauty et up was (Bredent, senden, Germany). Then a silicon key was done, which is transparent and made labially to allow light curing using visio.sil ILT (Visio.il ILT (Bredent, senden, Germany). The veneers were removed afterwards and cleaned thoroughly to remove any wax tracers and then returned to their positions in the silicon index. A special adhesive visio.link (visio.link (Bredent, senden, Germany) was applied on the BioHPP framework and the inner side of the veneers then light curing. Polymerization was achieved via 2 stages, in- termediate and final. Intermediate polymerization was done by a hand light cure unit for fixation of the layers. And final Polymerization was then done in a special UV curing unit (Bre.Lux Power unit Bredent, senden, Germany) for $180 \mathrm{sec}$. Finally, the BioHPP framework was finished by a tungsten carbide bur and polished with a goat-hair brush and acrypol or pumice. Fig(3)

\section{Strain gauge installation}

The strain gauges (kyowa strain gauges, Japan) used in this study had a length of $1 \mathrm{~mm}$, width $2.4 \mathrm{~mm}$ and nominal resistance $120 \mathrm{Ohm}$. The strain gauges were installed in their grooves distal to two posterior implants. The wires of the strain gauges were embedded in grooves created in the base of the model and fixed in position using bonding agent. The fixed detachable prosthesis to be tested were tightened into place following the manufacturer's recommendations.

The load applied with a plunger in midpoint of horizontal plate was increased from 0 to 100 $\mathrm{N}$ at a constant rate of $0.5 \mathrm{~mm} / \mathrm{min}$. After fifteen minutes the same load was applied unilaterally on the right side to represent the working side at the central groove of first molar using I bar shaped load applicator then after fifteen minutes cast was tilted 30 degree and load was applied obliquely. The process was repeated five times for each group and average of maximum induced strains in supporting structure were recorded and tabulated.

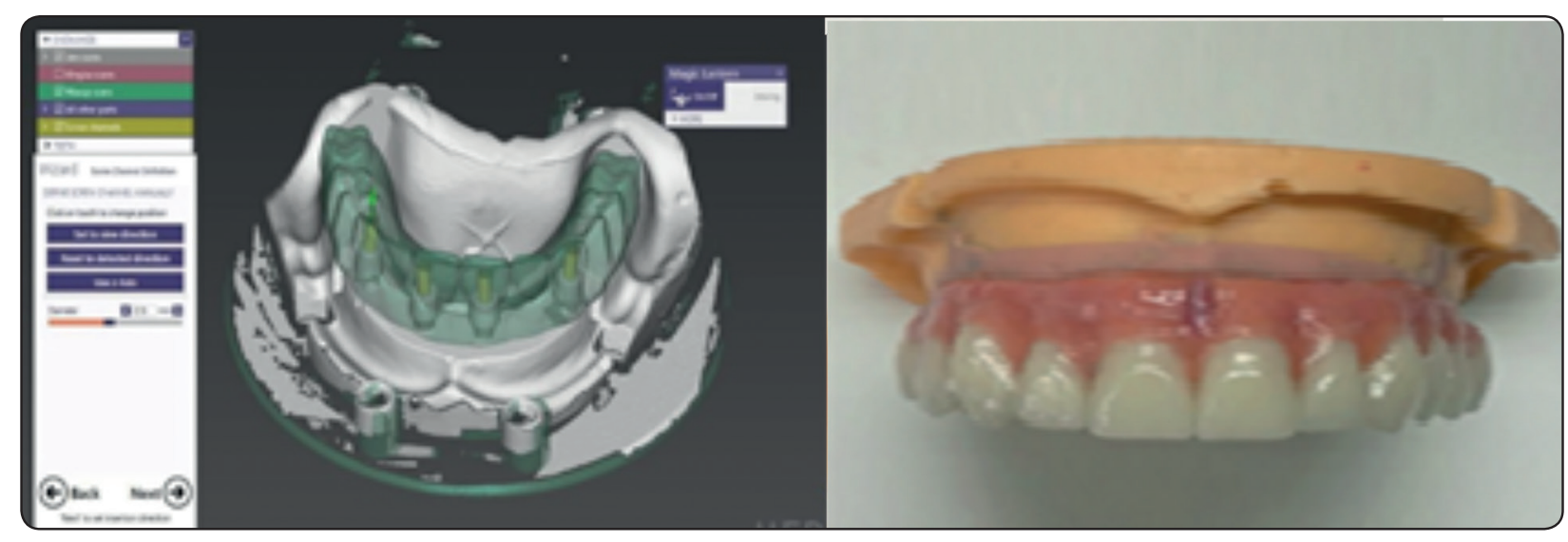

Fig. (3): Construction of BioHPP prosthesis. 


\section{RESULTS}

Statistical analysis was performed with SPSS 22 (SPSS, Inc., Chicago, IL, USA). Data were first presented as means and standard deviation values. $T$ test was used for comparison bet ween groups, at a significance level at $\mathrm{P} \leq 0.05$.

When a bilateral load was applied in a vertical direction on upper first molar area, it was found that the mean and standard deviation of bilateral microstrain fall on the implants retaining the chrome-cobalt fixed detachable prosthesis was $130.34 \mathrm{~N}$ while the mean of bilateral microstrain on the implants retaining the BioHPP fixed detachable prosthesis was $88.36 \mathrm{~N}$. Statistical analysis revealed statistical significant difference between chromecobalt \&BioHPP p value 0.06as shown in (Table.1)

When a vertical unilateral load was applied on the upper right first molar area, it was found that the mean of vertical unilateral microstrain on implants retaining chrome-cobalt denture was $210.74 \mathrm{~N}$ while it was found $160.29 \mathrm{~N}$ on the implants retaining BioHPP fixed detachable prosthesis. Statistical analysis revealed that the average vertical load falling on implants was more in chrome-cobalt group than BioHPP group and there was significant difference between them as shown in (Table.1)

When an oblique load was applied, it was found that the mean of stresses on the implants retaining chrome-coblat fixed detachable prosthesis was $180.64 \mathrm{~N}$ while it was $120.89 \mathrm{~N}$ on implants retaining BioHPP fixed detachable prosthesis. Statistical analysis revealed that the average oblique load falling on implants was more in chrome-cobalt than BioHPP and there was significant difference between them as shown in (Table. 1)

\section{DISCUSSION}

This study was carried out in vitro to allow for better control over variables and to facilitate measurements of changes which occur. In vitro study was carried out as it seemed beneficial in providing valid comparative data excluding the effect of variation in the tissues overlying the ridge and the form and quality of supporting ridge. ${ }^{(16)}$

The test model used in this study was fabricated utilizing 3D printing technology. This is justified due to the good accuracy of stereolithography technology. Rapid Prototyping technology has attracted enormous interest among researchers because it greatly facilitates the realization of threedimensional 3D objects as well as the speed of production with high accuracy. It was found that the lowest strain values of passivity of fit were recorded in Stereolithography fabricated prosthesis. Accuracy of 3D printed model might be attributed to the fact that they exhibit no or nil amount of internal stresses due to the mode of fabrication through building the cast layer by layer. ${ }^{(17)}$

The results obtained from this study showed that under bilateral loading, stresses delivered to the supporting implants under the fixed prosthesis was reduced and the load was distributed on the alveolar residual ridge and the implants in comparison with unilateral loadings, while under unilateral loadings

TABLE (1) Mean and std. deviations of stress induced on supporting structure of studied groups. p value of independent $t$ test

\begin{tabular}{|c|c|c|c|c|c|}
\hline & \multicolumn{2}{|c|}{ Group A } & \multicolumn{2}{c|}{ Group B } & \multirow{2}{*}{ P value } \\
\cline { 2 - 5 } & Mean & Std. deviation & Mean & Std. deviation & \\
\hline Bilateral Vertical loading & 130.34 & 25.7 & 88.36 & 18.45 & 0.016 \\
\hline Unilateral Vertical loading & 210.74 & 37.45 & 160.29 & 25.45 & 0.037 \\
\hline Oblique loading & 180.64 & 30.76 & 120.89 & 35.58 & 0.019 \\
\hline
\end{tabular}


the stresses were concentrated at the loaded implant and ridge in vertical load and at the loaded implant in oblique load.

This finding could be attributed to the wide distribution of forces over a square area under bilateral load involving more planes and to the favorable support achieved with the quadrilateral design and due to its potential to dissipate the stresses uniformly between both the ridge and the implants with its splinting effect. While under unilateral loadings, the rotational movement of the prosthesis concentrates the stresses at the loaded implants and the loaded ridge under unilateral vertical loading. ${ }^{(18)}$

Stresses induced in the supporting structures during occlusal loading (unilateral or bilateral) were lower in group with BioHPP frame work compared to chrome cobalt frame work. This may be explained by the fact that lower modulus of elasticity of frame work material which will absorbs more energy from the applied occlusal load, and transfers less energy to the supporting structures. BioHPP will absorb the occlusal forces, thus decreases its effect on the bone implant interface. ${ }^{(19)}$

Stress analysis revealed that the average load falling on implants in the loaded side was higher in chrome-cobalt subjected to oblique forces which may be attributed to that, oblique loads have been reported to increase stress values in peripheral bone and prosthetic components, and also generating great stress in the crown, implant, abutments, and cortical bone during mastication. Therefore, occlusal interferences must be eliminated, and an optimum occlusal relation should be established for long- term survival. ${ }^{(20)}$

\section{CONCLUSION}

Within the limitations of this study, it could be concluded that, unilateral loading was more traumatic to the implants as compared to bilateral loading. Biohpp framework has damping effect as it transfer less stress to supporting structures compared to chrome cobalt- acrylic framework.

\section{REFERENCES}

1. Malo P, Rangert B, Dvarsater L. Immediate function of Branemark implants in the esthetic zone: a retrospective clinical study with 6 months to 4 years of follow-up. Clin Implant Dent Relat Res. 2000;2:138- 46.

2. Malo P, Rangert B, 1Nobre M. "All-on-Four" immediatefunction concept with Branemark System implants for completely edentulous mandibles: a retrospective clinical study. Clin Implant Dent Relat Res. 2003;5:2-9.

3. Malo P, Friberg B, Polizzi G, Gualini F, Vighagen T, Rangert B. Immediate and early function of Branemark System implants placed in the esthetic zone: a 1-year prospective clinical multicenter study. Clin Implant Dent Relat Res. 2003;5:37-46.

4. Brånemark PI, Engstrand $\mathrm{P}$, Ohrnell LO, Gröndahl K, Nilsson P, Hagberg K, et al. Brånemark Novum: a new treatment concept for rehabilitation of the edentulous mandible. Preliminary results from a prospective clinical follow-up study. Clin Implant Dent Relat Res. 1999; $1: 2-16$.

5. Agliardi E, Clerico M, Ciancio P, Massironi D. Immediate loading of full-arch fixed prostheses supported by axial and tilted implants for the treatment of edentulous atrophic mandibles. Quintessence Int. 2010;41:285-93.

6. Agliardi EL, Pozzi A, Stappert CF, Benzi R, Romeo $\mathrm{D}$, Gherlone E. Immediate fixed rehabilitation of the edentulous maxilla: a prospective clinical and radiological study after 3 years of loading. Clin Implant Dent Relat Res. 2014;16:292-302.

7. Chrcanovic BR, Albrektsson T, Wennerberg A. Immediate nonfunctional versus immediate functional loading and dental implant failure rates: a systematic review and metaanalysis. J Dent. 2014;42:1052-9.

8. Chrcanovic BR, Albrektsson T, Wennerberg A. Immediately loaded non-submerged versus delayed loaded submerged dental implants: a meta-analysis. Int J Oral Maxillofac Surg. 2015;44:493-506.

9. M Taruna, B Chittaranjan, N Sudheer, Suchita Tella, Md. Abusaad, .PROSTHODONTIC PERSPECTIVE TO ALLON-4® CONCEPT FOR DENTAL IMPLANTS.Journal of Clinical and Diagnostic Research. 2014, 8(10): ZE16ZE19

10. Hassel AJ, Shahin R, Kreuter A, et al: Rehabilitation of an edentulous mandible with an implant-supported fixed 
prosthesis using an all-ceramic framework: a case report. Quintessence Int 2008;39:421-426

11. Bozini T, Petridis H, Garefis K: A meta-analysis of prosthodontic complication rates of implant-supported fixed dental prostheses in edentulous patients after an observation period of at least 5 years. Int J Oral Maxillofac Implants 2011;26:304-318

12. Linkevicius T, Vladimirovas E, Grybauskas S, et al: Veneer fracture in implant-supported metal-ceramic restorations. Part I: Overall success rate and impact of occlusal guidance. Stomatologija 2008;10:133-139

13. NAJEEB S, ZAFAR MS, KHURSHID Z, SIDDIQUI F, Applications of polyetheretherketone (PEEK) in oral implantology and prosthodontics. J Prosthodont Res. 2016 Jan ;60(1):12-9

14. Bechir, Edwin \& Bechir, A \& Cherana, Gioga \& Manu, Roxana \& Burcea, A \& Teodora Dascalu, Ionela. The Advantages of BioHPP Polymer as Superstructure Material in Oral Implantology. MATERIALE PLASTICE. 2016.53(3):394-398.
15. Assif D, Marshak B, Horowitz A. Analysis of load transfer and stress distribution by an implant-supported fixed partial denture. J Prosthet Dent. 1996;75(3):285-291.

16. Stansbury BE. A retentive attachment for overdentures. J Prosthet Dent. 1976;35(2):228-230.

17. Karl M, Wichmann MG, Heckmann SM, Krafft T. Strain development in 3-unit implant-supported CAD/ CAM restorations. Int J Oral Maxillofac Implants.2008; 23(4): 648-652.

18. Doundoulakis JH, Eckert SE, Lindquist CC, Jeffcoat MK. The implant- supported overdenture as an alternative to the complete mandibular denture. J Am Dent Assoc. 2003;134(11):1455-1458.

19. Menini M, Conserva E, Tealdo T, et al. Shock absorption capacity of restorative materials for dental implant prostheses: an in vitro study. Int J Prosthodont.2013; 26(6):549-556.

20. Morneburg TR, Pröschel PA. Measurement of masticatory forces and implant loads: a methodologic clinical study. Int J Prosthodont.2002; 15(1):20-27. 